\section{Report of the 2008 ASMS Fall Workshop: Protein-Protein and Protein-Ligand Interactions}

\author{
Organized by Julie A. Leary and Joseph A. Loo ${ }^{\mathrm{b}}$ \\ ${ }^{a}$ Department of Molecular and Cellular Biology and \\ Department of Chemistry, University of California, \\ Davis, Davis, California, USA \\ ${ }^{b}$ Department of Biological Chemistry and Department \\ of Chemistry and Biochemistry, University of \\ California, Los Angeles, Los Angeles, California, USA
}

A Workshop on the principles and practice of mass spectrometry for the study of "Protein-Protein and Protein-Ligand Interactions" was held on November 6-8, 2008 in the Financial District/Chinatown district of San Francisco, CA. Over 95 attendees listened to presentations from nine speakers discussing the important experimental protocols of applying electrospray ionization (ESI) with mass spectrometry (MS) and ion mobility spectrometry (IMS) for studying noncovalent protein complexes with small-molecule ligands, metals, carbohydrate, nucleic acids, and other proteins.

Each of the speakers discussed the experimental aspects for successful analysis of protein complexes, such as sample desalting and preparation, instrumental variables associated with the mass spectrometer and/or ion mobility instrument, and data analysis. Data from a number of different ESI-MS/IMS instruments were presented during the 2-day workshop, including time-of-flight (TOF), quadrupole TOF, Fourier transform ion cyclotron resonance (FT-ICR), traditional drift tube IMS, and Triwave IMS.

The first day of the Workshop featured presentations highlighting the unique advantages of IMS for studying the structures of small and large protein complexes. Julie Leary (University of California, Davis; "Analysis of Protein-Protein and Protein-Carbohydrate Complexes") discussed her group's research involving chemokine interactions with glycosaminoglycans using FTICR and the molecular architecture of the large $800-\mathrm{kDa}$ eukaryotic initiation factor (eif3) using Triwave IMS. Mike Bowers (University of California, Santa Barbara; "Characterization of Amyloid Proteins and Aggregation: Ion Mobility Theory and Practice) discussed the application of IMS for studying the aggregation behavior of Alzheimer's disease-related amyloid beta protein and Parkinson's disease-related $\alpha$-synuclein protein, and he presented higher-resolution ion mobility separation using a long drift tube. Brandon Ruotolo (Cambridge University; "Ion Mobility-Mass Spectrometry Analysis of Large Protein Complexes") presented a detailed discussion of how their laboratory tunes and calibrates the Waters Synapt IMS instrument for studying large protein complexes. Alison Ashcroft (University of Leeds; "Protein Assembly Monitored by MS: More Than Just Mass Measurements") discussed how a combination of hydrogen-deuterium exchange, tandem mass spectrometry, and IMS was used to elucidate the important structural aspects of $\beta_{2}$-microglobulin misfolding, leading to fibril formation and dialysisrelated amyloidosis.

A lively discussion ensued as to how one should measure and calculate collision cross sections and calibrate drift times for IMS, especially with the Triwavebased Synapt instrument. Several of the presenters discussed the pros and cons of their methods. Because of the growing popularity and utility of IMS-based measurement of collision cross section for large protein studies, it was acknowledged by most of the attendees that this subject will be the focus of more attention in the future.

The second day provided a broader range of subjects related to the direct measurement of noncovalent protein complexes. Joseph Loo (University of California, Los Angeles; "Instrumentation and Methods for Characterizing Protein-Drug and Protein-Metal Binding") discussed the advantages of using MS for measuring protein-ligand binding in the context of the pharmaceutical/biotechnology arena, and he discussed how MS/MS using QTOF and FT-ICR can be used to determine the sites of ligand binding. The analysis of very large viral protein complexes, including the 3- to 4-MDa capsid of the hepatitis B virus, using TOF, QTOF, and IMS, was demonstrated by Esther van Duijn-Keizers (Utrecht University; "Studying Functional Macromolecular Assemblies by Native Mass Spectrometry").

A detailed treatise on the application of ESI-MS for determining protein-ligand binding constants and for discerning nonspecific binding was presented by John Klassen (University of Alberta; "Quantifying ProteinLigand Interactions by the Direct ES-MS Assay"). Dan Fabris (University of Maryland-Baltimore County; "Conciliating the Extremes: MS Analysis of ProteinNucleic Acid Assemblies") introduced the experimental protocols specific to measuring protein-nucleic acid complexes, such as methods for reducing salt adducts. He demonstrated how MS and MS/MS could be used to determine sites of binding for protein-RNA complexes related to HIV.

The final presentation was delivered by Vicki Wysocki (University of Arizona; "Proteolysis, Crosslinking, CID and SID Methods for Probing Protein Complexes"). The talk featured her group's effort to develop surface-induced dissociation (SID) coupled with QTOF-MS for characterizing the structures of proteins and protein complexes. Preliminary data sug- 


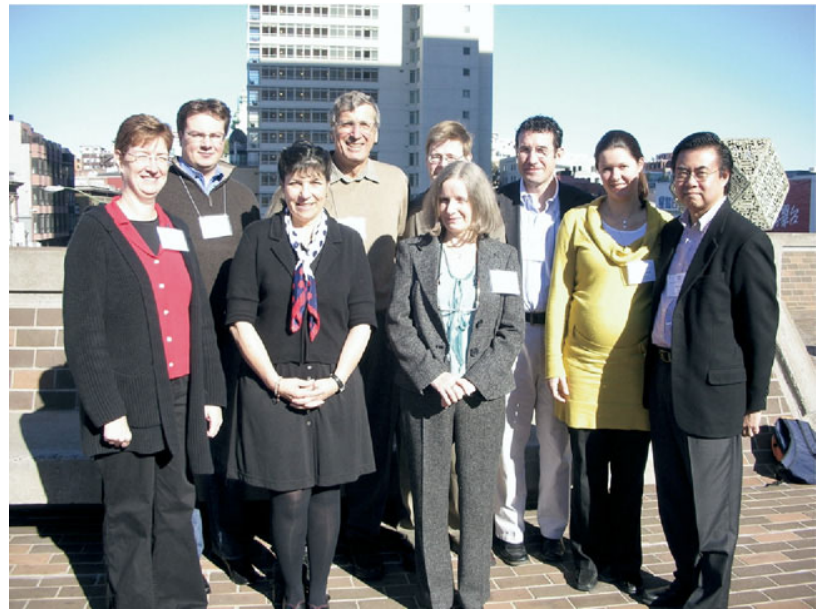

ASMS Fall Workshop speakers. Front row (left to right): Vicki Wysocki, Julie Leary, Alison Ashcroft. Back row: Brandon Ruotolo, Mike Bowers, John Klassen, Dan Fabris, Esther van DuijnKeizers, Joe Loo.

gest that SID might be a useful tool for revealing the architecture of large assemblies, such as the $800-\mathrm{kDa}$ GroEL complex.

As a new feature of the ASMS Workshop format, poster presentations were displayed throughout the 2-day meeting. Poster presenters were invited to give a brief, 5-min oral summary of their poster to the Workshop attendees. Those presenting include: Heather Bischel, Stanford University (Noncovalent Binding Interactions of Long-Chain Perlfuorochemicals with Serum Albumin); Anne Blackwell, University of Arizona
(Extension of Surface-Induced Dissociation to Heterogeneous Non-Covalent Protein Complexes); Iain Campuzano, Waters Corporation (Using Ion Mobility to Measure Changes in Protein Structure upon SubstrateLigand Binding); Eric Dodds, University of Arizona (Collision-Induced Dissociation and Surface-Induced Dissociation of Non-Intermingling and Domain-Swapped Homodimeric Protein Complexes); Shirley Lomeli, UCLA (Increasing Charge While Preserving Noncovalent Protein Complexes for ESI-MS); Pragya Singh, University of Washington (Characterization of Protein Complexes by Crosslinking and an "Open-Modification" Search Strategy); Ryan Wenzel, CovalX (Analysis of Intact High Mass Protein Interactions by MALDI Mass Spectrometry); Zhengping Yi, Arizona State University (Identification of Novel IRS-1 Binding Partners by HPLC-ESI-MS/MS).

The ASMS Fall Workshop is a venue for learning by fostering open discussions. The attendees took advantage of this atmosphere by raising many questions on a range of subjects, which included: use of commercial nanoelectrospray tips or home "pulled" tips, specific versus nonspecific interactions in the gas phase and whether the structure determined from gas-phase data accurately reflects the solution-phase conformation. Several attendees commented that the workshop discussions could have lasted a few more days. Attendance remained high throughout the workshop and the meeting adjourned late Friday afternoon, allowing attendees to depart for home or take in the San Francisco nightlife. 